\title{
. Dreikernige Transplantate zwischen Acetabularia crenulata und mediterranea I
}

\author{
Von JOACHIM HämMERLING \\ Aus dem Deutsch-Italienischen Institut für Meeresbiologie Rovigno, \\ z. Zt. Institut für Seenforschung und Seenbewirtschaftung der Kaiser-Wilhelm-Gesellschaft, \\ Langenargen a. B.
}

Meinem Lehrer Max Hartmann zum 70. Geburtstage gewidmet

(Z. Naturforschg. 1, 337-342 [1946]; eingegangen am 27. April 1946)

\begin{abstract}
An dreikernigen Transplantaten mit zwei Kernen von Acetabularia crenulata und einem Kern von $A$. mediterranea entstanden stark nach cren neigende Hüte. Da an zweikernigen T'ransplantaten mit einem cren- und einem med-Kern weniger stark nach cren neigende $\mathrm{Z}_{\mathrm{wi}} \mathrm{i}$ schenstufen entstanden, ist zu schließen, daß der Grad einer Zwischenstufe vom Mengenverhältnis der artverschiedenen Gestaltungsstoffe abhängt.
\end{abstract}

$\mathrm{W}$ Tenn in zweikernigen Transplantaten zwischen cren und med $\left(\text { cren }_{1} \text { med }_{1}\right)^{1}$ die kernabhängigen, artverschiedenen Hutgestaltungsstoffe gemeinsam wirken, so werden zwischen den beiden Arten stehende Hüte gebildet. In ihren Einzelmerkmalen sind sie verschieden stark abgelenkt, teils mehr nach med, teils mehr nach cren neigend, teils ungefähr in der Mitte stehend. Solche Hüte wur-

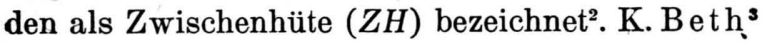
erhielt an zweikernigen Acic $_{1}$ med $_{1}$-Transplantaten die gleichen Ergebnisse. Inzwischen hat sich das früher mitgeteilte Material erhöht: an 92 Transplantaten entstanden $197 \mathrm{ZH}$ und nur 9 mehr oder weniger artreine cren- oder med-Hüte. Für die Ausnahmen ließ sich nachweisen oder wahrscheinlich machen, daß eine Störung im System vorlag, z.B. ein Kern ausgefallen war. An funktionstüchtigen zweikernigen Transplantaten entsteht also nur ein Huttyp, offenbar wegen ungefähr gleichbleibender Mischungsverhältnisse der artverschiedenen Gestaltungsstoffe. Wurden dagegen einkernige Transplantate zwischen den genannten Arten hergestellt - kernhaltiges Teilstück von med + kernloses Teilstück von cren oder Acic und umgekehrt - so entstanden verschiedene Typen von Hüten: entweder wieder $Z_{\text {wischenhüte oder }}$

1 Benutzte Symbole: cren = Acetabularia crenulata, med $=$ A. mediterranea, Acic $=$ Acicularia Schenckii. Das Suffix gibt die Zahl der von der betreffenden Art vorhandenen Kerne an, also $1=1 \mathrm{Kern}, 2=2$ Kerne, $0=$ kein Kern (kernloses Teilstück).

2 J. H äm th e r ling, Z. indukt. Abstammg.-Vererbgsl. 81, 114 [1943].

3 Z. indukt. Abstammg.-Vererbgsl. 81, 271 [1943].

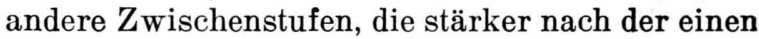
oder anderen Art tendierten und als $Z H \rightarrow$ med, $Z H \rightarrow$ cren und $Z H \rightarrow A c i c$ bezeichnet wurden, oder schließlich gleich kerngemäße Hüte. Alle Umstände sprachen dafür, daß den vom kernhaltigen Teilstück produzierten Stoffen verschiedene Mengen gespeicherter Stoffe im kernlosen Teilstück gegenüberstanden, daß also der Grad einer Zwischenstufe vom Mengenverhältnis der Hutgestaltungsstoffe abhängt ${ }^{4}$. .

Diese Deutung mußte sich auf einem anderen, direkteren Wege nachprüfen lassen: wenn in zweikernigen cren $_{1}$ med $_{1}$-Transplantaten ein bestimmtes Mengenverhältnis von cren- und med-Stoffen vorliegt, so sollte es sich in dreikernigen cren $_{2}$ med $_{1^{-}}$ Transplantaten mit 2 crẹn-Kernen und 1 med $d$-Kern zugunsten der cren-Stoffe verschieben, die ent-

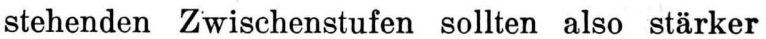
nach cren neigen als die der zweikernigen Transplantate. Voraussetzung hierfür ist allerdings, daß auch solche Transplantate noch keine wesentlichen Störungen erleiden. Diese Voraussetzung traf zu, und so konnte $1943^{2}$ bereits kurz mitgeteilt werden, daß unveröffentlichte Versuche von Wolf die Erwartung erfüllten: cren $_{2}$ med $_{1}$-Transplantate

- Die Speicherung von Formbildungsstoffen ging bereits aus den regenerativen Leistungen kernloser Teilstücke hervor; J.Hämmerling, Roux'Archiv 131, 1 [1934]; Naturwiss. 22, 829 [1934]. Nach den neueren Befunden2, 3 ist zwischen hut-bildenden und hut-gestaltenden Stoffen zu unterscheiden. In dieser Arbeit haben wir es nur mit den letzten zu tun, d. h. mit denjenigen Stoffen, deren Wirkung die Prägung der Artmerkmale zuzuschreiben ist. 
bildeten Zwischenhüte $\rightarrow$ cren. Inzwischen ist Frau Wolf das gesamte Material verloren gegangen, so daß eine Wiederholung nötig war. Da Frau Wolf diese nicht ausführen konnte, wurden die Versuche von mir wiederholt.

Von med wurden Pflanzen einer $\boldsymbol{F}_{2}$ vom 5. Januar 1944 benutzt, von cren Pflanzen der Kulturen $F_{3+4}$ vom 9 . Juni und 12. Juni 1943 (beide hatten ganz oder fast dieselbe Herkunft). Die Herstellung dreikerniger Transplantate stieß auf keine Schwierigkeiten. 15 Transplantate wurden zunächst als cren $_{1}$ med $_{1}$-Transplantate angelegt, drei weitere als rren $_{2}$-Transplantate, und zwar, wie üblich, durch Ineinanderschieben der Stiele kernhaltiger Teilstücke (Näheres Fußn. 2). Das aus der Verwachsungsstelle ausgewachsene Regenerat wurde nach passender Zeit (3-8 Wochen) zurückgeschnitten und auf den verbleibenden Stumpf das dritte kernhaltige Teilstück verpflanzt (Abb.1). Aus der neuen Verwachsungsstelle wuchsen dann ein, mitunter auch zwei Regenerate aus; in einigen Fällen trieb zusätzlich ein Rhizoidast aus.

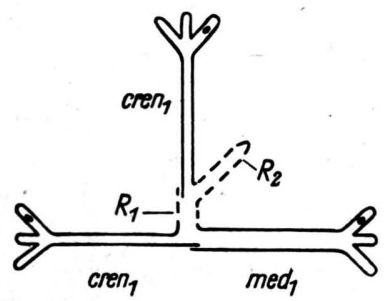

Abb. 1.

Transplantationsschema. Rhizoide der drei Komponenten durch drei Äste angedeutet, Kern = schwarzer Punkt in ihnen. $R_{\mathbf{1}}=$ zurückgeschnittener Stumpf des aus den zuerst transplantierten Teilstücken entstandenen Regenerates, $R_{2}=$ das aus der neuen Verwachsungsstelle entstehende Regenerat.
Ein Transplantat wurde so angelegt, daß ein cren $_{1}$ med $_{1-}$ Transplantat hergestellt und gleichzeitig auf einen überstehenden Ast des med-Rhizoides das zweite cren-Stück verpflanzt wurde. Insgesamt wurden also 19 Dreier hergestellt.

Zunächst sind die Ergebnisse an den verschiedenen Kontrollen der benutzten Kulturen zu besprechen. Die Ausgangskulturen selbst waren in bestem Zustand. Weiter wurden 18 cren $_{1^{-}}, 18$ cren $_{2^{-}}, 14$ med $_{1^{-}}$und 14 med $_{2^{-}}$Teile geprüft (z. Tl. aus Versuchen Maschlankas stammend).Diese wurden zwar nicht alle bis zur Hutbildung gehalten, soweit dieses aber der Fall war, verhielten sie sich hierin wie auch in allen anderen regenerativen Eigenschaften normal, d.h. die ein- und zweikernigen cren-Teile bildeten normale cren-Hüte, die med-Teile normale med-Hüte. Ferner wurden 18 cren $_{0}$ med $_{1}$-Transplantate hergestellt (also kernloses $c r e n$ - + kernhaltiges medTeilstück), ebenso 12 cren $_{1}$ med $_{1}$-Transplantate, die alle bis zur Hutbildung gehalten wurden. In bezug auf Häufigkeit und Grad der entstehenden $\mathrm{Z}$ wischenstufen waren keine Abweichungen von den früheren Ergebnissen festzustellen. Diese Prüfung war aus einem speziellen Grunde wichtig. Die benutzte med-Kultur verhielt sich zwar in den morphologischen Hutmerkmalen normal, besaß aber neben reichlicher Wirtelbildung wahrscheinlich erhöhte Wuchsgeschwindigkeit. Es war daher denkbar, daß ihre Hutgestaltungsstoffe erhöhte Wirkungskraft besäßen, was sich an den Zwischenstufen der cren med $_{1}$ - und cren med $_{1}$-Transplantate hätte äußern müssen. Das war aber, wie eben geschildert, nicht der Fall. Daher müssen auch die an den dreikernigen cren $_{2}$ med $_{1}$-Transplantaten entstandenen $\mathrm{Z}_{\mathrm{w}}$ ischenstufen, $\mathrm{ZH} \rightarrow$ cren, als die für diese Kombination normale Zwischenstufe betrachtet werden. Dies geht auch aus folgenden, besonders demonstrativen Kontrollversuchen hervor: bei 5 cren $_{1}$ med $_{1}$ - und 2 cren $_{2}$-Transplantaten, die später zu Dreiern gemacht wurden, wurde mit der Antransplantation des dritten Teiles so lange gewartet, bis sie einen oder zwei Hüte gebildet hatten. Diese waren, wie

\begin{tabular}{|c|c|c|c|c|c|}
\hline & med & $Z h$ & $\mathrm{Zh} \longrightarrow$ cren & cren & \\
\hline Hutanlage & anders als cren & $\begin{array}{l}\text { cren-ähnlich, aber Primär- } \\
\text { anlagen oft rerschoben } \\
\text { und ebenso die auswach- } \\
\text { senden Kammern }\end{array}$ & $\begin{array}{l}\text { noch cren-ähnlicher als die } \\
Z h \text {, da Verschiebungen } \\
\text { geringer oder fehlend }\end{array}$ & $\begin{array}{l}\text { keine Verschie- } \\
\text { bungen }\end{array}$ & Abb. 4 \\
\hline $\begin{array}{l}\text { Kammer- } \\
\text { ende }\end{array}$ & ohne Sporn & rund, selten etwas spitz & oft spitz oder Spornansatz & $\begin{array}{l}\text { mit Sporn oder } \\
\text { spitz }\end{array}$ & Abb. 2,3 \\
\hline $\begin{array}{l}\text { Kammer- } \\
\text { form }\end{array}$ & Keil & schlank & schlank oder keulig & keulig & Abb. 2,3 \\
\hline $\begin{array}{l}\text { Kammer- } \\
\text { verbindung }\end{array}$ & verwachsen & $\begin{array}{l}\text { von Anfang an unverbun- } \\
\text { den (wegen Verschie- } \\
\text { bungen in Anlage) }\end{array}$ & öfter cren-artig verbunden & $\begin{array}{l}\text { verbunden, spä- } \\
\text { ter öfter isoliert } \\
\text { durch Kammer- } \\
\text { trennung }\end{array}$ & Abb. 2 \\
\hline $\begin{array}{l}\text { Haargröße } \\
\text { auf cor. sup. }\end{array}$ & sehr klein & klein, aber größer als med & $\begin{array}{l}\text { größer als } Z h \text {, fraglich ob } \\
\text { schon ebenso groß wie cren }\end{array}$ & am größten & $\begin{array}{l}\mathrm{Abb} \\
2 \mathrm{a}, 2 \mathrm{c}\end{array}$ \\
\hline
\end{tabular}

Tab. 1

Überblick über die bislang geprüften Hutmerkmale der $Z h \rightarrow$ cren im Vergleich mit den artreinen Formen und ihren Zwischenhüten 
zu erwarten, im ersten Falle Zwischenhüte, im zweiten Falle cren-Hüte. Die Dreier bildeten dagegen wieder $\mathrm{ZH} \rightarrow$ cren.

Um nun zu den Ergebnissen an den Dreiern zu kommen, so blieb von den 19 cren $_{2}$ med $_{1}$-Transplantaten nur eines ohne Hüte, und zwar ein erkranktes Transplantat. Die Hutbildungsfähigkeit war also trotz Vorhandenseins dreier Kerne gegenüber ein- und zweikernigen Teilen in keiner Weise beschränkt. Wie bei den zweikernigen cren $_{1}$ med $_{1}$ Transplantaten entstanden mehrere Hüte, wobei aber wieder fraglich bleibt, ob sich in dieser Hinsicht die cren-Eigenschaften durchsetzten (cren bildet mehrere, med nur einen Hut; näheres Fußn. 2).

Insgesamt wurden von den 18 hutbildenden Transplantaten 62 Hüte gebildet, von denen der größte Teil, nämlich $46, Z H \rightarrow$ cren waren (Tab.2). 44 dieser Hüte waren ganz oder annähérnd typisch. Der Bau, solcher Hüte ist bereits $1943^{2}$ geschildert wor-

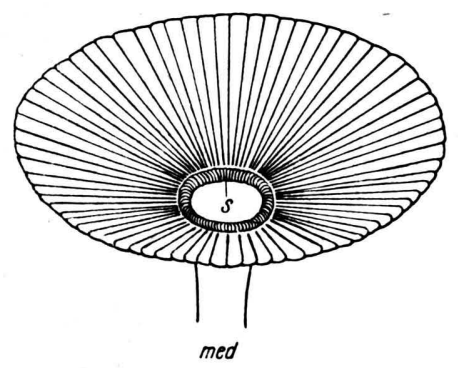

Abb. 2 a.

med-Hut. 72 Kammern, verwachsen. In der Mitte die corona superior $(s)$, Haare bei dieser Vergrößerung nicht erkennbar. Etwa $6 \times$.

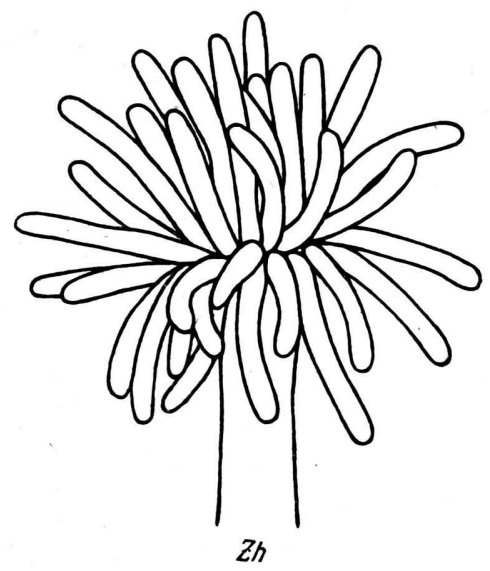

Abb. 2 b.

Zwischenhut eines zweikernigen cren $_{1}$ med $_{1}$-Transplantates. Kammern isoliert, schlank, ohne Sporn, vorne abgerundet, nur 33 Kammern gezeichnet. Etwa $15 \times$.

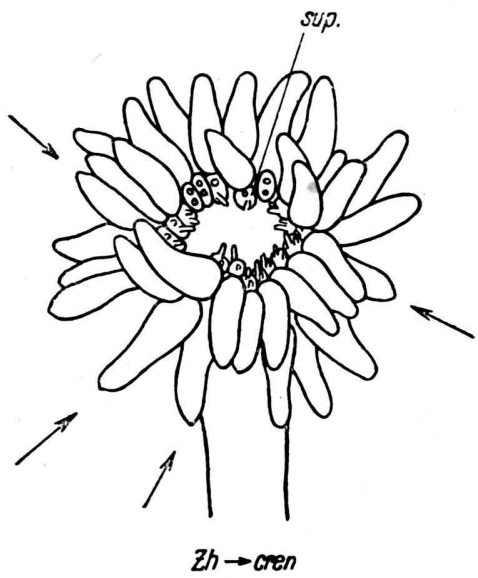

Abb. 2 c.

$\mathrm{Zw}$ ischenhut $\rightarrow$ cren eines dreikernigen cren $_{2}$ med $_{1-}$ Transplantates. 34 Kammern, z. Tl. verbunden, z. Tl. vorn spitz (Pfeile: in Wirklichkeit spitzer, als Abb. wiedergibt), Kammerform stark nach cren gehend. sup = corona superior mit je zwei Haarstummeln, der innere länger, Haargröße etwa wie cren. Etwa $16 \times$.

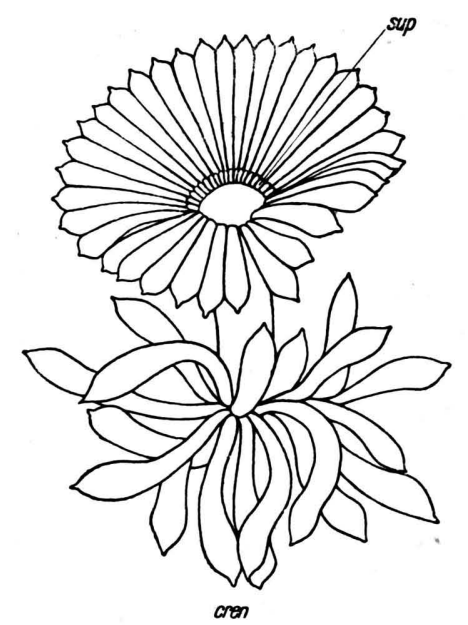

Abb. $2 d$

Zwei übereinanderstehende cren-Hüte, am unteren Hut Kammern isoliert (nur 18 vorn liegende Kammern gezeichnet), am oberen noch weitgehend verbunden, $35 \mathrm{Kammern}$. K. gespornt. sup = corona superior (an sich sichtbare Haare nicht gezeichnet. Etwa 9,5X.

den. (Diese waren zwar an med $_{0}$ cren $_{1}$-Transplantaten entstanden, doch ist das unwesentlich.) Dort sind auch die Einzelmerkmale der artreinen crenund med-Hüte sowie der $\mathrm{Z}$ wischenhüte eingehend dargestellt worden. Wir können uns daher an dieser Stelle mit dem in Tab. 1 wiedergegebenen kurzen Überblick sowie dem Hinweis auf die Abb. 2-4 begnügen. Die $Z H \rightarrow$ cren wurden noch nicht in allen Eigenschaften untersucht, vor allem noch nicht quantitativ. Daß sie stärker nach cren neigen 
als die $Z H$, geht aber aus folgenden Eigenschaften hervor: das Kammerende war häufig mehr oder weniger zugespitzt, ja eine ganze Reihe von Kammern trug einen, wenn auch kleinen knopfartigen Sporn (Abb.2c, 3). Die Kammerform war entweder noch schlank wie bei den zweikernigen $\mathrm{Zwischen-}$ hüten oder schon keulig wie die von artreinen cren-Kammern (Abb. 2c, 3). Die Primäranlagen waren meist weniger oder gar nicht mehr verschoben (Abb. 4). Als Folge blieben einzelne Kammergruppen oder gar alle Kammern eines Hutes oft längere Zeit cren-artig verbunden (Abb.2c), so daß der Habitus eines cren-Hutes ziemlich erreicht war. Auch in der Größe der auf der Corona superior stehenden Haare war ein stärkerer crenEinfluß als bei $\mathrm{Zwischenhüten} \mathrm{deutlich} \mathrm{(vergl.} \mathrm{Ab-}$

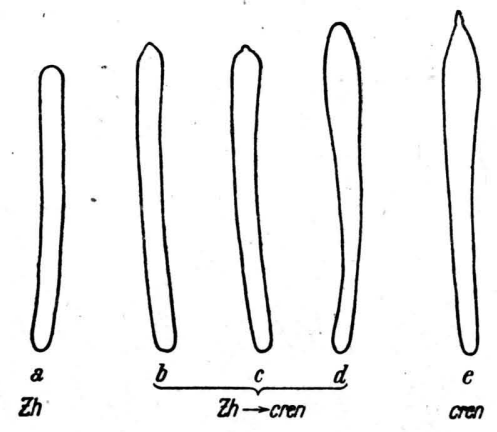

Abb. 3.

a) isolierte Kammern eines cren $_{1}$ med $_{1}-\mathrm{Z}$ wischenhutes, schlank, ohne Sporn.

b-d) desgl. eines cren ${ }_{2}$ med $_{1}-\mathrm{Zw}_{\text {wischenhutes } \rightarrow \text { cren. }}$. b) vorn spitz, c) mit Spornansatz, d) ohne Sporn, aber Form wie cren. e) cren-Kammer mit Sporn (Coronae inferiores und superiores nicht mitgezeichnet). a-e etwa $15 X$.

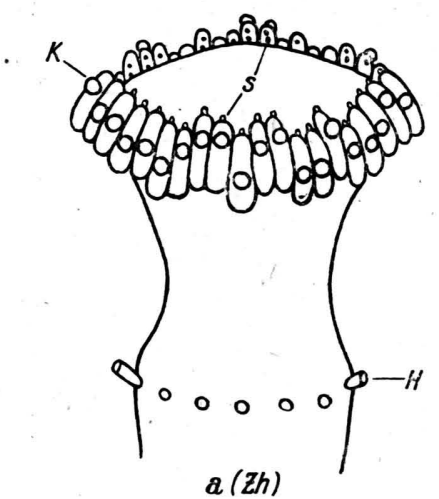

Abb. 4 a.

Zwischenhut eines zweikernigen cren $_{1}$ med $_{1}$-Transplantates in Anlage. Primäranlagen z. Tl. gegeneinander verschoben, Kammeranlagen $(K)$ auf diesen in verschiedener Höhe auswachsend. sup.-Haare $(s)$ sehr klein. Etwa $50 \times$.

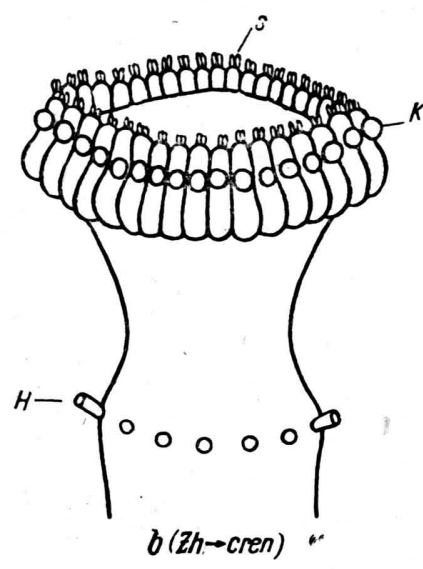

Abb. 4 b.

$Z h \rightarrow$ cren eines dreikernigen cren $_{2}$ med $_{1}$-Transplantates, ebenfalls in Anlage. Primär- und Kammeranlagen $(K)$ in gleicher Höhe, sup.-Haare größer $(s ;$ diese stehen hintereinander, nicht wie es die Abb. wiedergibt, nebeneinander). Dieser Hut ist von einem artreinen cren-Hut gleichen Stadiums nicht zu unterscheiden. $H=$ abgeschnittene Haare des letzten Wirtels. a, b etwa $50 \times$, stark schematisiert.

bildung 2c). Nehmen wir zu diesem Ergebnis hinzu, daß die von Frau Wolf untersuchten Dreier - mindestens 15 mit mindestens 30 Hüten - ebenfalls überwiegend oder nur $Z H \rightarrow$ cren bildeten, so bestätigt sich, daß der Grad einer Zwischenstufe von dem Mengenverhältnis der artverschiedenen Gestaltungsstoffe abhängt.

\begin{tabular}{|c|c|c|c|}
\hline $4 Z h$ & $\begin{array}{l}3 Z h \stackrel{\mathrm{w}}{\rightarrow} \text { cren } 33 Z h \stackrel{\mathrm{n}}{\rightarrow} \text { cren } \\
9 \mathrm{\text {st }} \rightarrow \text { cren } 1 Z h \rightarrow \text { cren } *\end{array}$ & $46 Z h \rightarrow$ cren & $\begin{array}{c}12 \text { undef. } \\
\text { Hüte }\end{array}$ \\
\hline
\end{tabular}

Tab. 2. Von 19 cren $_{2}$ med $_{1}$-Transplantaten blieb 1 ohne Hüte. Die übrigen 18 bildeten 62 Hüte, und zwar:

$\mathrm{w}=$ wenig nach cren, $\mathrm{n}=$,normal" nach cren, st $=$ stark nach cren neigend, immer aber mehr nach cren als zweikernige $\mathrm{Zh}$.

* Atypischer Hut, Stärkegrad nicht bestimmbar.

Diesem allgemeinen Ergebnis sind folgende Einzelheiten hinzuzufügen (Tab. 2) : Dem Habitus nach ließen sich die $Z H \rightarrow$ cren in drei Gruppen einteilen. Die am häufigsten auftretende wurde als Normaltypus bezeichnet (33 Hüte an 14 Transplantaten, Abb. 2c) ${ }^{\mathbf{5}}$.

9 Hüte (an 5 Transplantaten) waren deutlich stärker nach cren abgelenkt. Bei diesen war in 7 Fällen mit funktionsschwacher med-Komponente zu rechnen; im achten Fall schien es aus besonderen Gründen möglich, daß die Stoffmischung zu Gunsten der cren-Stoffe verschoben war. Nur im neunten Falle bot sich keine spe-

5 Hiermit soll nicht gesagt sein, daß an den 14 Tr. nur $\mathrm{ZH}$ cren auftraten. Das gilt auch für die folgenden Transplantate. An demselben Transplantat können vielmehr gelegentlich verschiedene Huttypen entstehen, wofür anschließend einige Beispiele angeführt sind. 
zielle Erklärungsmöglichkeit; vorher hatte das Transplantat jedoch zwei normal nach cren abgelenkte Hüte gebildet.

3 Hüte (an 3 Transplantaten) waren merklich schwächer als der Normaltypus nach cren abgelenkt. Hier war in einem Falle wieder mit abweichender Stoffmischung zu rechnen, dieses Mal zu Ungunsten der cren-Stoffe. Im zweiten Falle waren 2 Hüte vorausgegangen, die noch normal, aber doch schon etwas schwächer abgelenkt waren, was vielleicht an einer gewissen Schwäche der einen cren-Mutterpflanze liegen könnte, deren Spornbildungstendenz sehr gering ausgeprägt war. Im dritten Falle schließlich wurde nur ein Hut, und zwar ein stark atypischer Hut, gebildet. Es läßt sich also in jedem Einzelfalle mehr oder weniger wahrscheinlich machen; daß die Abweichung vom Normaltypus des $\mathrm{ZH} \rightarrow$ cren durch besondere Umstände bedingt war, ja in den meisten Fällen gibt es Erklärungsmöglichkeiten, warum die Ablenkung gerade in der eingeschlagenen Richtung ging. Auch wenn andere Gründe vorliegen sollten, so würden aus der Tatsache einer relativ großen Variabilität natürlich keine Bedenken gegen die Annahme quantitativer Abhängigkeitsverhältnisse entstehen, handelt es sich doch stets ưm $\mathrm{ZH} \rightarrow$ cren.

Ebensowenig geben die wirklichen Ausnahmen, nämlich $4 \mathrm{Zwischenhüte} \mathrm{(2} \mathrm{typische,} 2$ atypische), die an 4 Transplantaten entstanden, zu Bedenken Anlaß. Bei 2 Transplantaten entstand hinterher je ein $\mathrm{ZH} \rightarrow$ cren, bei dem dritten waren vorher (an 2 Regeneraten) sogar $6 \mathrm{ZH} \rightarrow$ cren entstanden. Die Entstehung der Zwischenhüte muß also besondere Gründe gehabt haben. Im vierten Falle dürfte es sich in Wirklichkeit um ein cren $_{0}$ med $_{\mathbf{1}^{-}}$ Transplantat gehandelt haben, für welche ja die Bildung von $\mathrm{Zwischenhüten} \mathrm{charakteristisch} \mathrm{ist}{ }^{2}$. Denn nach Zerlegung in die Ausgangskomponenten blieben beide cren-Stücke ohne Regenerat, und nur das med-Stück regenerierte. Im übrigen verhielt sich dieses Transplantat allgemein atypisch, indem ein krankes Regenerat mit nur einem mißbildeten Hut entstand. Schließlich entstanden wie an den ein- und zweikernigen Systemen nicht auswachsende, undefinierbar bleibende Kümmerhüte, und zwar 12 an 10 Transplantaten. Diese bildeten außerdem alle mindestens einen $\mathrm{Zh} \rightarrow$ cren.

Nach dem Ergebnis an den cren $_{2}$ med $_{1}$-Transplantaten durfte erwartet werden, daß an der reziproken Kombination mit 1 cren- und 2 med-Kernen $\left(\right.$ cren $_{1}$ med $_{2}$ ) nach med abgelenkte $\mathrm{Zwischenstufen}$ entstehen würden. Dieser Versuch wurde von H. Maschlanka ${ }^{6}$ ausgeführt. Dabei zeigte sich aber, daß eine wesentliche Voraussetzung nicht zutraf, so daß schon aus diesem Grunde verständlich wird, wenn die Erwartung nicht erfüllt wurde. Diese Dreier wiesen nämlich nach anfänglich normaler Regeneration meist starke Störungen auf und bildeten nur selten Hüte. Nach dem Ergebnis an den rren $_{2}$ med $_{1}$-Transplantaten kann das nicht

\footnotetext{
6 Biol. Zbl. (im Druck).
}

an der Dreikernigkeit als solcher, sondern muß an der spezifischen Kernkombination liegen. Unter denjenigen Transplantaten, die es bis zur Hutbildung brachten, waren weiterhin nur drei, deren Kerne als voll funktionstüchtig angesehen werden konnten. Sie bildeten je einen Hut. Diese drei Hüte waren ihrem Habitus nach $\mathrm{Z}$ wischenhüte, also nicht stärker nach med abgelenkt. Bei so kleiner Zahl läßt sich aber natürlich nicht erkennen, ob es sich um eine Regel handelt, es bleibt vielmehr abzuwarten, welche Verhältnisse sich bei größerem Material herausstellen werden. Hiervon abgesehen besteht ein gewisser Verdacht, daß zur Entstehung stärker nach med abgelenkter $\mathrm{Z}_{\text {wischenstufen, die }}$ auch äußerlich sogleich als solche erkennbar sind, also von $Z H \rightarrow$ med, das Stoffverhältnis noch stärker nach med verschoben sein muß, als es in den drei cren $_{1}$ med $_{2}$-Transplantaten der Fall war. Es entstanden nämlich an cren $_{1}$ med $_{2}$-Transplantaten solche Hüte, aber anscheinend nur, wenn der crenKern geschädigt war. Wie sich die Dinge auch aufklären mögen, im Widerspruch zur Annahme quantitativer Abhängigkeitsverhältnisse stehen die cren med $_{2}$-Transplantate nicht. Denn diese Annahme ist ja nicht nur durch die cren $_{2}$ med $_{1}$-Transplantate, sondern auch durch die einkernigen Transplantate gut gestützt, und zwar sowohl in den cren med-als in den Acic med-Kombinationen.

In diesem Zusammenhange sei auf eine noch ungeklärte Frage hingewiesen. Die Stoffproduktion der reinen Arten muß in einem bestimmten, im einzelnen freilich unbekannten Verhältnis stehen. Schon in den zweikernigen cren $_{1}$ med $_{1}$-Transplantaten braucht es nicht dasselbe zu bleiben. Es könnten vielmehr Beeinflussungen stattfinden. Nehmen wir an, es herrsche dort das Verhältnis $1 \mathrm{a}: 1 \mathrm{~b}$, wobei $\mathrm{a} \gtreqless \mathrm{b}$ sein könnte, so folgt hieraus nicht, daß es in dreikernigen cren $_{2}$ med $_{1}$-Transplantaten auf das Verhältnis $2 \mathrm{a}: 1 \mathrm{~b}$ anwachsen muß, sondern die a-Menge sollte zwar größer als 1 , könnte aber auch kleiner als 2 sein. Ebenso brauchte in cren med $_{2}$-Transplantaten nicht das Verhältnis $1 \mathrm{a}: 2 \mathrm{~b}$ zu herrschen. Ein anderes Verhältnis wäre gerade bei dieser Kombination mit ihren häufigen Störungen nicht überraschend. Erwarten läßt sich also in dreikernigen Transplantaten nur eine Verschiebung des Stoffverhältnisses in bestimmterRichtung, und auch nur, wenn im sonstigen Verhalten keine Störungen zu bemerken sind, die über die an ein- und zweikernigen Systemen vorhandenen hinausgehen. Der Grad der Verschiebung dagegen 
bleibt zunächst unbekannt. Ebensowenig läßt sich voraussagen, welche $\mathrm{Zwischenstufe}$ bei einer bestimmten Verschiebung des Stoffverhältnisses gebildet werden wird. Um ein konkretes Beispiel zu

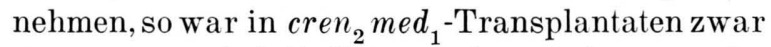
zu erwarten, daß die Hüte stärker nach cren neigten als die der cren $_{1}$ med $_{1}$-Transplantate, aber sie hätten durchschnittlich auch weniger oder stärker nach cren neigen können, als es tatsächlich der Fall war.

Es muß versucht werden, die näheren Ursachen der Hutlosigkeit der cren $_{1}$ med $_{2}$-Transplantate und, soweit möglich, auch die Frage des Grades der
Stoffverschiebung an vierkernigen Kombinationen weiter zu klären, also an cren $_{3}$ med $_{1^{-}}$, cren $_{1}$ med $_{3}-$ und cren $_{2}$ med $_{2}$-Transplantaten. Für die letzten liegen bereits unveröffentlichte Versuche von Maschlanka vor, aus denen sich mit großer Wahrscheinlichkeit ergibt, daß auch diese Kombination nicht zur Hutbildung fähig ist, obwohl die Kernzahl in demselben Verhältnis wie bei cren $_{1}$ med $_{1}$-Transplantaten steht. An der Vierkernigkeit als solcher kann das wiederum nicht liegen, da vierkernige artreine rren $_{4}$ - und med $_{4}^{-}$ Transplantate Hüte bildeten, anscheinend ohne Schwierigkeit.

\title{
Die Züchtung von Polyploiden mit positivem Selektionswert
}

\author{
Von Josef Straub
}

Aus dem Kaiser-Wilhelm-Institut für Biologie, Abteilung v on W ettste in, Hechingen-Boll

(Z. Naturforschg. 1, 342-345 [1946]; eingegangen am 29. März 1946)

\begin{abstract}
Der Bastard aus Antirrhinum majus Sippe 50 und Antirrhinum glutinosum Wildsippe orgiva ist durch Heterosis und volle Fertilität gekennzeichnet. Er wird tetraploid gemacht. In den diploiden und tetraploiden Nachkommenschaften werden jeweils die wüchsigsten und fertilsten Typen selektioniert. Die stärkste Heterosis tritt in einzelnen Linien der tetraploiden Nachkommenschaften auf. Die wüchsigsten tetraploiden Linien stellen gegenüber den diploiden auch Frühblüher dar. Das Auftreten der frühblühenden Heterosistypen steht im Gegensatz zu allen Befunden an Tetraploiden aus reinen Linien, denn diese sind durch eine Verzögerung der Gesamtentwicklung charakterisiert. In den tetraploiden Nachkommenschaften läßt sich auch die ursprünglich niedere Fertilität durch Selektion erhöhen. Damit ist die Methode aufgezeigt, wie man Polyploide mit positivem Selektionswert auslösen kann. Es ist hierzu notwendig, daß Bastarde, deren Eltern sich in einer großen Zahl von Genen unterscheiden, der Polyploidisierung unterworfen werden. Doch braucht die Verschiedenheit der Bastardeltern nicht so extrem zu sein, daß dadurch im diploiden Bastard Sterilitätserscheinungen bedingt sind.
\end{abstract}

$\mathrm{D}$ ie Wirkung der Genomvermehrung auf die morphologischen und physiologischen Eigenschaften der Pflanzen ist eingehend untersucht worden. Die Grundlage für die morphologischen Anderungen ist durch das erhöhte Zellvolumen gegeben. Vergrößerte Organe folgen daraus. Die „Gigastypen“ haben jedoch eine verringerte Zellteilungsrate, ihre Stoffproduktion ist nicht erhöht, ihr osmotischer Wert erweist sich in der Mehrzahl der untersuchten Fälle als gesenkt. Angesichts dieser Eigenschaften der künstlich ausgelösten Polyploiden bleibt die erhöhte Anpassungsfähigkeit der natürlichen Polyploiden und die gegenüber den

1 J. Straub, Biol. Zbl. 60, 559 [1940].

2 F.von Wettstein, Z. ind. Abst.- u. Vererbungslehre 74, 35 [1937]. diploiden Wildarten gesteigerte Leistung der polyploiden Kulturpflanzen unverständlich. Einige qualitative Änderungen, die als Folge der quantitativen auftreten, vermögen den künstlich ausgelösten Autopolyploiden zwar Vorteile zu verschaffen ${ }^{1}$, doch fallen sie sicher nicht ins Gewicht. Schließlich wirkt noch die Fertilitätssenkung, die bei der Polyploidisierung mehr oder minder reiner Arten stets eintritt, als ein starker negativer Faktor. Man könnte dagegen einwenden, daß sekundäre Genmutationen die alten Fertilitätsverhältnisse wiederherzustellen vermögen ${ }^{23}$. Aber dieser Vorgang

${ }^{3}$ F. von Wettstein, Ber. dtsch. bot. Ges. 58, 374 [1940].

${ }_{4}$ F.von Wettstein u. J.Straub, Z. ind. Abst.u. Vererbungslehre 80, 271 [1942]. 